\title{
CONHECIMENTO DE MANIPULADORES DE ALIMENTOS SOBRE HIGIENE PESSOAL E BOAS PRÁTICAS NA PRODUÇÃO DE ALIMENTOS
}

\section{KNOWLEDGE OF FOOD HANDLERS ABOUT PERSONAL HYGIENE AND GOOD PRACTICES IN FOOD PRODUCTION}

\author{
Luara Thabata Alves Boaventura ${ }^{1}$ \\ Liliane Pereira Frades ${ }^{2}$ \\ Márcia Lopes Weber ${ }^{3}$ \\ Bruna Oliveira Silva Pinto ${ }^{4}$
}

\begin{abstract}
Resumo: As refeições produzidas em Unidades de Alimentação e Nutrição-UAN escolares devem atender às necessidades nutricionais dos alunos, oferecendo-lhes, além de alimentos adequados nos aspectos sensoriais e nutricionais, produtos seguros em relação às condições higiênico-sanitárias. Boas práticas de manipulação de alimentos são necessárias para garantir a produção e oferta de alimentos seguros. $O$ objetivo deste estudo foi identificar o nível de conhecimento sobre boas práticas de manipuladores de alimentos de UAN, de uma comunidade situada em São Paulo/SP, antes e após a aplicação de treinamento. O método utilizado foi um instrumento proposto na literatura, aplicado antes e após treinamento sobre o tema. Esse instrumento era composto de 10 questões sobre higiene e boas práticas na manipulação de alimentos, envolvendo lavagem das mãos, higiene pessoal e contaminação dos alimentos. O treinamento foi desenvolvido em quatro encontros. A primeira aplicação do questionário foi efetuada um mês antes do treinamento, e a segunda um mês após o último encontro. Os resultados obtidos evidenciaram que as respostas corretas sobre boas práticas na manipulação dos alimentos apresentaram similaridade, antes e após aplicação de treinamento. Entre os temas que apresentaram incremento nas respostas corretas, constavam descarte de óleo, higienização de utensilios, manejo de resíduos e contaminação dos alimentos. Palavras-chave: Manipulação de alimentos; vigilância sanitária; boas práticas de manipulação; segurança de alimentos.
\end{abstract}

Abstract: Meals produced in school restaurants (UAN) must meet the nutritional needs of the students, offering them adequate food in the sensory and nutritional aspects and safe food in terms of hygienicsanitary conditions. Good practices are needed to ensure the preparation and offer of safe food. The objective of this study was to identify the level of knowledge about good practices of UAN food handlers in a low-income community in São Paulo, Brazil, before and after training. We used a quiz proposed in the literature, applied before and after training. The quiz consisted of 10 questions about hygiene and good practices in food handling, involving hand washing, personal hygiene and food contamination. The training was developed in four meetings. The first application of the questionnaire was one month before the first training, and the second one month after the last training meeting. The results showed that the correct answers about good practices in food handling presented similarity, before and after the application of training. Among all topics that presented increase in the correct answers were oil discarding, utensil hygiene, waste destination and food contamination.

Keywords: Food handlers; sanitary surveillance; good practices; food safety.

\footnotetext{
1 Acadêmica do curso de Nutrição - Centro Universitário Adventista de São Paulo, SP, Brasil. E-mail: luaraboaventura@hotmail.com.

2 Acadêmica do curso de Nutrição - Centro Universitário Adventista de São Paulo, SP, Brasil. E-mail: lili_frade@hotmail.com.

3 Nutricionista, docente nos cursos de graduação da Universidade Anhembi Morumbi e Centro Universitário Adventista de São Paulo, SP, Brasil. E-mail: marciaws@yahoo.com.br.

4 Acadêmica do curso de Nutrição - Centro Universitário Adventista de São Paulo, SP, Brasil. E-mail: vrboliveira@gmail.com.
} 


\section{INTRODUÇÃO}

As refeições produzidas em Unidades de Alimentação e Nutrição-UAN escolares devem atender às necessidades nutricionais dos alunos, oferecendo-Ihes, além de alimentos adequados aos aspectos sensorial e nutricional, produtos seguros em relação às condições higiênico-sanitárias, protegendo e promovendo a saúde dos beneficiários (CARDOSO et al., 2010).

As condições higiênico-sanitárias inadequadas dos alimentos produzidos em cozinhas escolares estão relacionadas a fatores importantes, como processo de produção desses alimentos, contaminação cruzada, higienização incorreta de alimentos, ambiente e manipuladores, conservação imprópria, e utilização de sobras armazenadas incorretamente. A influência desses fatores na qualidade sanitária dos alimentos ocorre porque eles proporcionam contaminação e proliferação de microorganismos, podendo contribuir para o aumento da ocorrência de doenças transmitidas por alimentos-DTA (SANTOS; BEZERRA, 2015). No Brasil, do total de surtos de DTA notificados, no período de 1999 a 2008, 10,7\% ocorreram em instituições de ensino (GOMES; CAMPOS; MONEGO, 2012). Entre as possíveis causas para a ocorrência dessas enfermidades, estão as práticas inadequadas de higiene e o processamento de alimentos por pessoas inabilitadas (OLIVEIRA; BRASIL; TADDEI, 2008). Os cuidados higiênicos no processo produtivo e a educação dos manipuladores envolvidos na preparação, processamento e processos são limites fundamentais para a prevenção da maioria das DTA (AGUIAR; KRAEMER, 2010).

Assim, a higiene e segurança alimentar em estabelecimentos que manipulam alimentos visa garantir uma adequada condição higiênico-sanitária dos produtos elaborados, sem oferecer riscos à saúde do consumidor, sob condições previstas pela legislação vigente (BRASIL, 2004). O conhecimento dos principais pontos de contaminação durante o processamento dos alimentos é essencial para garantir qualidade microbiológica e segurança para o consumidor. As boas práticas de higiene e manipulação e a educação continuada dos manipuladores de alimentos contribuem para a redução da incidência de DTA (MARMETINI et al, 2010).

A partir disso, é preciso orientar e treinar os manipuladores sobre os cuidados na aquisição, acondicionamento, manipulação, conservação e exposição ao consumo dos alimentos, bem como a estrutura física do local de manipulação, para que a qualidade sanitária do alimento não esteja em risco pelos perigos químicos, físicos e biológicos que oferecem. Dessa forma, as boas práticas de manipulação são regras que, quando praticadas, ajudam a reduzir os perigos ou contaminação de alimentos (MARMETINI; RONQUI; ALVARENGA, 2010).

Assim, este trabalho teve como objetivo identificar o nível de conhecimento sobre boas práticas de manipuladores de alimentos de UAN de uma comunidade em São Paulo/SP, antes e após a aplicação de treinamento. 


\section{MÉTODO}

O estudo integrou o projeto de extensão Programa de Capacitação Continuada em Higiene na Manipulação de Alimentos, desenvolvido por um centro universitário junto a uma comunidade, situada na zona sul de São Paulo/SP. Esse programa é uma resposta à demanda local de apoio à capacitação de manipuladores de alimentos em centros de apoio à produção e distribuição de refeições na comunidade, e ocorreu ao longo de 2016. Participaram do estudo manipuladores de três UAN da comunidade, sendo duas em creches e uma em centro de produção de lanches para comercialização, geridos por uma associação sem fins lucrativos.

Os 17 manipuladores dos locais envolvidos foram convidados a participar do estudo. Sua participação foi voluntária, e assim que o manipulador concordou em participar, recebeu e assinou um Termo de Consentimento Livre e Esclarecido-TCLE, em duas vias, contendo a descrição dos objetivos e métodos da pesquisa, assim como o contato dos pesquisadores. $O$ trabalho foi aprovado pelo Comitê de Ética em Pesquisa do Centro Universitário Adventista de São Paulo/UNASP, sob protocolo 44566115300005377.

Os conteúdos abordados no treinamento foram baseados nas recomendações da legislação sanitária (BRASIL, 2002; BRASIL, 2004), e a capacitação consistiu na apresentação teórico-prática de informações com foco nos temas higiene pessoal, de alimentos e ambiental. Foram realizados quatro encontros de uma hora cada um, com intervalos de um mês entre os encontros. Cada tema proposto foi abordado em um encontro, e, no último encontro, foi realizada retomada das informações abordadas nos encontros anteriores. Os conteúdos foram abordados por meio da apresentação de slides, aplicação de demonstrações, dinâmicas e simulações de situações características da produção de alimentos. Ao longo da apresentação, a participação dos manipuladores foi estimulada por intermédio de perguntas dirigidas. Ao final de cada encontro, foram aplicadas avaliações dos conteúdos abordados por meio da distribuição de figuras de cozinhas em operação e manipuladores com erros para serem identificados, alguns quiz, para serem respondidos individualmente e em grupos, e discussão dirigida sobre as boas práticas abordadas e efetivamente adotadas pelos participantes. Também foram distribuídos folhetos explicativos contendo as informações tratadas em abordagem clara e por meio de figuras. Todo o material foi elaborado pelas autoras.

O método utilizado para a identificação do nível de conhecimento dos manipuladores sobre higiene pessoal e boas práticas na produção de alimentos envolveu a aplicação de um questionário. Esse instrumento foi utilizado por Colombo, Oliveira e Silva (2009) em pesquisa similar com merendeiras de creches e escolas de Santa Fé/PR, e era composto de 10 questões relativas a conhecimentos sobre higiene e boas práticas na manipulação de alimentos. O questionário foi aplicado individualmente, em sala reservada, no próprio local de trabalho, e em dois momentos, 
antes e após a aplicação de treinamento de capacitação em higiene na manipulação de alimentos.

A primeira aplicação do questionário foi efetuada um mês antes do primeiro encontro para capacitação, e a segunda, um mês após o último encontro para capacitação. O conteúdo das questões do instrumento foi definido por Colombo, Oliveira e Silva (2009), a partir das recomendações presentes na legislação sanitária, e as alternativas de respostas foram apresentadas de maneira a serem classificadas em conforme, e não conforme em relação à adequação proposta pela mesma legislação (BRASIL, 2002; SÃO PAULO, 2011). Para classificação do nível de conhecimento dos manipuladores, foi calculado o percentual de acertos das respostas a partir da conformidade delas à legislação, conforme parâmetros propostos por Mello et al (2010) e Jorge et al (2013): conhecimento adequado - 76 a $100 \%$ de acertos; conhecimento regular - 51 a $75 \%$ de acertos; e conhecimento deficiente - 0 a $50 \%$ de acertos.

Para a comparação das respostas consideradas corretas antes e após o treinamento, foi calculado o teste $\mathrm{T}$ de Student, considerando como diferença estatisticamente significante $p \leq 0,05$.

\section{RESULTADOS E DISCUSSÃO}

Os participantes do estudo em sua maioria foram mulheres (76\%). Quanto ao nível de escolaridade, $47 \%$ possuíam o ensino médio completo. Esses resultados são superiores aos observados por Silva, Germano e Germano (2003), que realizou uma pesquisa com indivíduos responsáveis pela merenda escolar de 24 unidades do ensino fundamental, localizadas em São Paulo/SP, onde revelou que, dos manipuladores de alimentos, apenas $12,5 \%$ haviam concluído o ensino médio.

A distribuição total de respostas corretas, antes e após o treinamento, apresentou similaridade, com $41 \%$ e 52\%, respectivamente (Tabela1). Observou-se aumento no número de respostas corretas após o treinamento nos temas: contaminação dos alimentos ( $8 \%$ versus $57 \%$ ), higienização de utensílios ( $29 \%$ versus $71 \%$ ), descarte de óleo $(76 \%$ versus $100 \%$ ) e manejo de resíduos (41\% versus $57 \%)$. Antes do treinamento, o percentual de acertos foi classificado como conhecimento deficiente (41\%) e após foi classificado como regular (52\%). Houve incremento de $11 \%$ no conhecimento demonstrado da primeira para a segunda aplicação do questionário, embora sem diferença estatisticamente significante $(p=0,93377)$.

Em relação à lavagem das mãos, as respostas foram similares antes e após 0 treinamento, com $53 \%$ e $57 \%$ de respostas corretas, respectivamente. Estudo semelhante sobre condições higiênico-sanitárias das cozinhas de creches públicas revelou que $100 \%$ dos manipuladores apresentaram inadequação no item técnica de higiene das mãos (SANTOS; BEZERRA, 2015). Ribeiro, Reis e Rossi (2000) ressaltam a relevância da lavagem das mãos como principal medida para reduzir a quantidade de 
micro-organismos, melhorando a segurança alimentar. Assim, a assimilação e adoção deste procedimento por manipuladores aumenta a segurança dos alimentos preparados.

Observou-se menor número de respostas corretas após o treinamento em relação à higiene pessoal ( $47 \%$ antes e $0 \%$ após). Estudo realizado em cozinhas escolares do município de Vitória da Conquista mostrou que mais da metade $(52,4 \%)$ dos profissionais não tinha hábitos higiênicos adequados no local de trabalho (OLIVEIRA; BRASIL, TADDEI, 2008). A presença e o uso adequado de lavatórios no setor de manipulação dos alimentos são importantes, considerando que higiene frequente $\mathrm{e}$ correta das mãos, juntamente com higiene pessoal dos manipulados de alimentos, são fundamentais à qualidade da alimentação servida (FONSECA et al, 2010).

Tabela 1. Distribuição das respostas dos manipuladores de alimentos em boas práticas, antes e após treinamento. São Paulo, 2016.

\begin{tabular}{|c|c|c|c|c|c|}
\hline \multirow[b]{2}{*}{ Tema } & \multicolumn{2}{|c|}{$\begin{array}{l}\text { Antes do Treinamento } \\
\qquad(\mathrm{n}=17)\end{array}$} & \multicolumn{2}{|c|}{$\begin{array}{l}\text { Após o Treinamento } \\
\qquad(\mathrm{n}=14)\end{array}$} & \multirow{2}{*}{$p$} \\
\hline & $\begin{array}{c}\text { Incorretas } \\
\mathrm{n} / \%\end{array}$ & $\begin{array}{c}\text { Corretas } \\
\mathrm{n} / \%\end{array}$ & $\begin{array}{c}\text { Incorretas } \\
\mathrm{n} / \%\end{array}$ & $\begin{array}{l}\text { Correta } \\
\text { s } \mathrm{n} / \%\end{array}$ & \\
\hline Lavagem das Mãos & $8 / 47$ & $9 / 53$ & $6 / 43$ & $8 / 57$ & \\
\hline Higiene Pessoal & $9 / 53$ & $8 / 47$ & $14 / 100$ & $0 / 0$ & \\
\hline $\begin{array}{l}\text { Contaminação dos } \\
\text { Alimentos }\end{array}$ & $14 / 82$ & $3 / 18$ & $6 / 43$ & $8 / 57$ & \\
\hline $\begin{array}{l}\text { Higienização dos } \\
\text { Folhosos }\end{array}$ & $9 / 53$ & $8 / 47$ & $6 / 43$ & $8 / 57$ & \\
\hline $\begin{array}{l}\text { Higienização dos } \\
\text { Utensílios }\end{array}$ & $12 / 71$ & $5 / 29$ & $4 / 29$ & $10 / 71$ & \\
\hline Utilização de Ovos & $11 / 65$ & $6 / 35$ & $10 / 71$ & $4 / 29$ & \\
\hline Descarte de óleo & $4 / 23$ & $13 / 76$ & $0 / 0$ & $14 / 100$ & \\
\hline Manejo de resíduos & $10 / 59$ & $7 / 41$ & $6 / 43$ & $8 / 57$ & \\
\hline $\begin{array}{l}\text { Prevenção de } \\
\text { vetores e pragas }\end{array}$ & $13 / 76$ & $6 / 35$ & $8 / 57$ & $6 / 43$ & \\
\hline Total & $90 / 56$ & $63 / 41$ & $60 / 48$ & $66 / 52$ & 0,93377 \\
\hline
\end{tabular}

Em duas das três UAN do presente estudo, havia presença de lavatórios na área de preparo das refeições. No entanto, a inexistência de respostas corretas sobre higiene de mãos após o treinamento reforça que, além da necessidade de instalação de lavatórios em todos os locais em estudo, é imprescindível o treinamento dos manipuladores em relação aos procedimentos adequados a esse procedimento. 
Sobre a contaminação dos alimentos, as respostas corretas após o treinamento aumentaram de $18 \%$ para $57 \%$. Resultado similar foi obtido por Colombo, Oliveira e Silva (2009), que observaram $56 \%$ das respostas das merendeiras nas creches e escolas em estudo corretas em relação à contaminação de alimentos. Práticas que geram contaminação cruzada e o próprio papel do manipulador na contaminação dos alimentos expõem as refeições produzidas a risco sanitário e de desenvolvimento de DTA pelos usuários. Ações preventivas durante o processo produtivo dos alimentos costumam ser eficazes para evitar a contaminação dos alimentos (FONSECA et al, 2010).

Diante dos resultados sobre higienização dos folhosos, houve aumento de acertos, de $47 \%$ para $57 \%$ respectivamente antes e após o treinamento. Um estudo realizado por Santos e Bezerra (2015) em cozinhas escolares do Município de Vitoria da Conquista/BA, revelou que $66,7 \%$ das escolas não realizam a higienização dos folhosos de forma adequada. Comparando os resultados sobre higienização de utensílios, houve aumento das respostas corretas após o treinamento, com $71 \%$ de acertos. Oliveira, Brasil e Taddei (2008), em estudo similar, observou $80 \%$ de inadequação na higienização dos utensílios.

Observou-se resultado maior sobre utilização de ovos, $29 \%$ a $35 \%$ de acertos, antes e depois, respectivamente. As merendeiras do estudo de Colombo, Oliveira e Silva (2009) apresentaram $48 \%$ de acertos nas questões envolvendo manipulação e higiene de ovos, com resultado superior ao do presente estudo. Uma das principais preocupações em relação aos procedimentos de manipulação envolvendo ovos referese ao risco de contaminação por Salmonella. Essa bactéria esteve presente como agente etiológico causador de surtos de DTA, no Paraná, em 81,49\% das ocorrências, de 2005 a 2008, e em 18,09\% das ocorrências, no mesmo Estado, de 2009 a 2014. Neste último período, a população de 5 a 14 anos representou $21 \%$ dos casos (GABARON; OTUTUMI; PIAU JÚNIOR, 2015).

O número de respostas corretas sobre descarte de óleo apresentou aumento após o treinamento, de $76 \%$ para $100 \%$ de acertos. Em estudo sobre o descarte de óleo, relativo ao descarte do óleo utilizado em frituras, todos os entrevistados responderam que o óleo usado era acondicionado em recipiente apropriado e destinado à reciclagem, e $67 \%$ dos entrevistados responderam que este processo era realizado por uma empresa terceirizada, especializada (BATISTA; FERNANDES; LESSA, 2010). Esta é a recomendação presente na legislação sanitária do município de São Paulo (SÃO PAULO, 2011).

Quanto ao manejo de lixo, houve aumento nas respostas corretas, após o treinamento, de $41 \%$ para $57 \%$. Estudo realizado por Oliveira, Brasil e Taddei (2008) mostrou que $20 \%$ das creches estudadas apresentavam acondicionamento do lixo de forma errada. O manejo do lixo de forma inadequada é uma situação que favorece a proliferação de vetores, como insetos e roedores, e consequente contaminação dos 
alimentos preparados (FONSECA et al., 2010).

Houve similaridade nas respostas relacionadas à prevenção de pragas e vetores, de $35 \%$ para $43 \%$, antes e após treinamento. Estudo realizado por Silva, Silva e Silva (2012) evidenciou que, em $83,3 \%$ das UAN, de seis escolas estaduais do município de Passos/MG, não se adotava nenhum tipo de medida preventiva e corretiva para evitar a atração de vetores e pragas, como instalação de telas em janelas e portas. A inexistência do controle de pragas e vetores pode acarretar prejuízos para a segurança microbiológica do alimento (FONSECA et al., 2010).

Como já mencionado, após o treinamento, houve incremento nas respostas corretas dos manipuladores em vários temas, com significativo aumento no tocante à contaminação de alimentos e descarte de óleo, embora sem diferença estatisticamente significante. Estudo realizado por Ghisleni e Basso (2008), que ministraram treinamento e verificaram grau de conhecimento de manipuladores de UAN em Santa Maria, RS, concluiu que logo após o treinamento a maioria dos manipuladores apresentou conhecimento classificado como muito bom ou bom (67\%), percentual maior que o do presente estudo, embora não houvesse parâmetro para comparar com respostas anteriores à capacitação. A pesquisa citada, de Ghisleni e Basso (2008), não mencionou o intervalo de tempo entre os dois encontros efetuados para o treinamento, mas utilizou métodos similares aos do presente estudo, com adoção de apresentação teórica de conteúdos, dinâmicas e demonstrações, também dando ênfase a atividades lúdicas. As atividades lúdicas são essenciais para introduzir e/ou reforçar os conceitos relacionados à microbiologia, como perigos, alimentos seguros e boas práticas, e costumam apresentar boa aceitação pelos participantes (BALLESTERO-ALVAREZ, 2001, apud LEITE et al, 2011). Isso foi observado no presente estudo, durante a aplicação da capacitação e, também, mencionado por Ghisleni e Basso (2008), embora não tenha sido possível estabelecer relação com os resultados do nível de conhecimento dos manipuladores.

Mesmo não tendo sido observada diferença estatisticamente significante entre as respostas corretas dos manipuladores antes e após o treinamento, recomenda-se que programas de capacitação sejam implementados nas UAN em estudo. Considerando a relevância dos manipuladores para a promoção e a garantia da produção de alimentos seguros, princípios de higiene pessoal e de alimentos devem ser continuamente reforçados e monitorados, sendo essencial a realização de atividades de educação e formação inicial e continuada (LEITE et al., 2011). A presença de manipuladores com conhecimento deficiente ou regular acerca de boas práticas de manipulação, conforme observado, não é uma realidade exclusiva das UAN do presente estudo, conforme foi apresentado na menção a pesquisas similares. Fonseca et al. (2010), em estudo relacionando as condições físico-funcionais de restaurantes comerciais à implementação das boas práticas em Minas Gerais, observaram que os problemas mais frequentes constatados no que referia-se à higienização foram a inexistência de manipuladores capacitados. A orientação continuada, por meio de treinamentos e de 
palestras sobre higiene em todas as etapas da produção de refeições, contribui para a fixação de conhecimentos e a constante reciclagem dos funcionários envolvidos na manipulação de alimentos (COLOMBO; OLIVEIRA; SILVA, 2009). No entanto, o fato de que o incremento nas respostas corretas após o programa de treinamento realizado neste estudo não apresentou diferença estatisticamente significante após os encontros de capacitação pode representar evidência de que a escolha dos métodos e/ou linguagem adotados poderiam ter sido mais criteriosos e eventualmente mais adequados ao grau de compreensão dos participantes.

\section{CONCLUSÃO}

Por intermédio dos resultados obtidos, pode-se verificar que as respostas corretas sobre boas práticas na manipulação dos alimentos apresentaram similaridade, antes e após aplicação de treinamento. Comparando as duas etapas do programa, houve alguns temas com incremento no nível de conformidade nas respostas, e outros, com queda no número de respostas corretas. Entre os temas que apresentaram incremento nas respostas corretas, constavam descarte de óleo, higienização de utensílios, manejo de resíduos e contaminação dos alimentos.

Sugere-se a implantação de programas de capacitação contínua de higiene e boas práticas de manipulação em todos os locais em estudo, garantindo reforço nas informações continuamente. Sugere-se, ainda, supervisão adequada no processo produtivo, garantindo refeições produzidas dentro de condições higiênico-sanitárias adequadas e consequente segurança aos seus usuários.

\section{REFERÊNCIAS}

AGUIAR, O.B.; KRAEMER, F.B. Educação formal, informal e não-formal na qualificação profissional dos trabalhadores de alimentação coletiva. Nutrire, v. 35, n. 3, p. 87-96, 2010.

BATISTA, A.P.A.; FERNANDES, L.T.M.; LESSA, N.M.V. Avaliação da quantidade de óleos e gorduras utilizados na preparação do cardápio semanal de uma Unidade Produtora de Alimentos (UPA), o impacto à saúde do comensal e verificação o descarte adequado ao meio ambiente. Nutrir Gerais, Ipatinga, v. 4, n. 7, p. 624-636, 2010.

BRASIL. Ministério da Saúde. Agência Nacional da Vigilância Sanitária- ANVISA. Resolução-RDC $n^{\circ}$ 216, de 15 de setembro de 2004. Dispõe sobre o regulamento técnico de boas práticas para serviços de alimentação. Diário Oficial da União, Brasília, 2004.. Disponível em:

<http://portal.anvisa.gov.br/documents/33916/388704/RESOLU\%25C3\%2587\%25C3 $\% 25830$ -

RDC\%2BN\%2B216\%2BDE\%2B15\%2BDE\%2BSETEMBRO\%2BDE\%2B2004.pdf/237 01496-925d-4d4d-99aa-9d479b316c4b> Acesso em: 25 set. 2015. Ministério da Saúde. Secretaria de Vigilância Sanitária. Resolução - RDC

n.275, de 21 out. 2002. Regulamento técnico de Procedimentos Operacionais 
Padronizados aplicados aos Estabelecimentos Produtores/Industrializadores de Alimentos e a Lista de Verificação das Boas práticas de Fabricação em Estabelecimentos Produtores/ Industrializadores de Alimentos. Diário Oficial da União, Brasília, 2002. Disponível em:

<http://www.rio.rj.gov.br/dlstatic/10112/5125403/4132350/ResoluuoRDC27521.10.200 2.pdf>. Acesso em: 25 set. 2015.

CARDOSO, R. C. V. et al. Avaliação da qualidade microbiológica de alimentos prontos para consumo servidos em escolas atendidas pelo Programa Nacional de Alimentação Escolar. Revista Instituto Adolfo Lutz, São Paulo, v. 69, n 2, p. 208-213, 2010.

COLOMBO, M.; OLIVEIRA, K. M. P; SILVA, D. L. D. Conhecimento das merendeiras de Santa Fé, PR, sobre higiene e boas práticas de fabricação na elaboração de alimentos. Higiene Alimentar, São Paulo, v. 23, n. 170, p. 39-46, 2009.

FONSECA, M. P. et al. Avaliação das condições físico-funcionais de restaurantes comerciais para implementação de boas práticas. Alimentos e Nutrição, Araraquara, v.21, n.2, p. 251-257, 2010.

GABARON, D.A.; OTUTUMI, L. K.; PIAU JÚNIOR, R. Surtos de salmonelose notificados no período de janeiro a julho de 2014 no Estado do Paraná, Brasil. Arq. Ciênc. Vet. Zool., Umuarama, v. 18, n. 1, p. 33-37, 2015.

GHISLENI, D.R.; BASSO, C. Educação em saúde a manipuladores de duas Unidades de Alimentação e Nutrição do município de Santa Maria/RS. Disciplinarum Scientia, Santa Maria, v. 9, n.1, p. 101-108, 2008.

GOMES, N. A. A. A.; CAMPOS, M. R. H.; MONEGO, E. T. Aspectos higiênicosanitários no processo produtivo dos alimentos em escolas públicas do Estado de Goiás, Brasil. Revista de Nutrição, Campinas, v.25, n.4, p.473-485, 2012.

JORGE, M. N. et al. Fatores relacionados aos conhecimentos de manipuladores de alimentos sobre boas práticas de manipulação em estabelecimentos comerciais.

Nutrir Gerais, Ipatinga, v. 7, n. 12, p. 1015-1029, 2013.

LEITE, C. L. et al. Formação para merendeiras: uma proposta metodológica aplicada em escolas estaduais atendidas pelo programa nacional de alimentação escolar, em Salvador, Bahia. Revista de Nutrição, Campinas, v. 24, n. 2, p. 275-285, 2011.

MARMETINI, P. R.; RONQUI, L.; ALVARENGA, O. V. A importância das boas praticas de manipulação para os estabelecimentos que manipulam alimentos. Revista Cientifica Facimed, p. 263-273, 2010. Disponível em: <http://www.facimed.edu.br/o/revista/pdfs/8770b901b3aff4febc857ec524d8cb40.pdf> Acesso em: 15 abr. 2015.

MELLO, G. A. et al. Conhecimentos dos manipuladores de alimentos sobre boas práticas nos restaurantes públicos populares do Estado do Rio de Janeiro. Brazilian Journal Food Technology, Campinas, v. 13, n. 1, p. 60-6, 2010.

OLIVEIRA, N. M.; BRASIL, D. L. A.; TADDEI, C. A. A. J. Avaliação das condições higiênico sanitárias das cozinhas de creches publicas e filantrópicas. Ciência e Saúde Coletiva Rio de Janeiro, v. 13, n. 3, p. 1051-1060, 2008. 
RIBEIRO, A. C.; REIS, D. O.; ROSSI, D. A. Procedimentos de higienização das mãos de manipuladores, em uma indústria frigorífica. Higiene Alimentar, São Paulo, v. 14, n. 70, p. 52-57, 2000.

SANTOS, P. G. I.; BEZERRA, M. V. Segurança de alimentos em cozinhas escolares do Munícipio de Vitoria da Conquista, Bahia. Revista Baiana de Saúde Pública, Salvador, v.39, n.2, p. 205-224, 2015.

SÃO PAULO. Secretaria Municipal de Saúde. Portaria $n^{\circ}$ 2619, de 06/12/2011.

Regulamento de Boas Práticas e de Controle de condições sanitárias e técnicas das atividades relacionadas à importação, exportação, extração, produção, manipulação, beneficiamento, acondicionamento, transporte, armazenamento, distribuição, embalagem, reembalagem, fracionamento, comercialização e uso de alimentos, águas minerais e de fontes, bebidas, aditivos e embalagens para alimentos. Disponível em: <http://www.prefeitura.sp.gov.br/cidade/secretarias/upload/chamadas/portaria_2619_1 323696514.pdf>Acesso em: 25 set. 2015.

SILVA, C.; GERMANO, M. I. S.; GERMANO, P. M. L. Condições higiênico-sanitárias dos locais de preparação da merenda escolar, da rede estadual de ensino em São Paulo/SP. Higiene Alimentar, São Paulo, v. 17, n.110, p.49-55, 2003.

SILVA, L.P.; SILVA, S.C.; SILVA, Q.R. Análise das boas práticas de fabricação de alimentos em cozinhas de escolas estaduais de Passos - MG: da escolha do produto ate o seu reaproveitamento. Revista Ciência Et Praxis, Belo Horizonte, v. 5, n. 9, p. 7-12, 2012. 